\title{
Effect of Aggregation on the Optical and Charge Transport Properties of an Anionic Conjugated Polyelectrolyte
}

\author{
Andres Garcia and Thuc-Quyen Nguyen \\ Mitsubishi Chemical Center for Advanced Materials, Department of Chemistry and \\ Biochemistry, University of California, Santa Barbara, CA 93106 \\ Corresponding author: quyen@chem.ucsb.edu
}

\section{Supporting Material}

Figure S1. Contact AFM topographic (a) and current image (b) of 95:5 (w:w) P3PBT:PEG blend film with $-500 \mathrm{mV}$ applied bias.

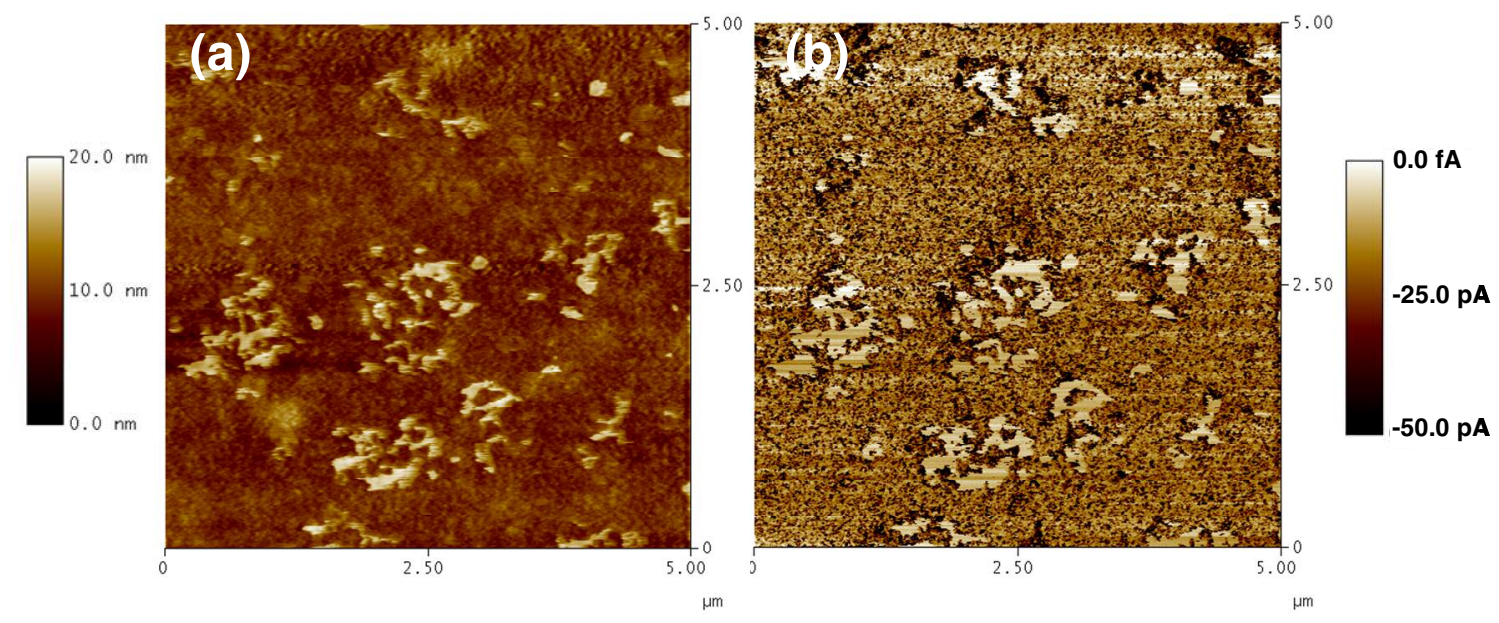


Figure S2. Current density versus bias characteristics of ITO/MEH-PPV/Au diode with and without pulsed bias scans. Pulse bias scans were performed with 500ms off times (0V) and 5ms on times.

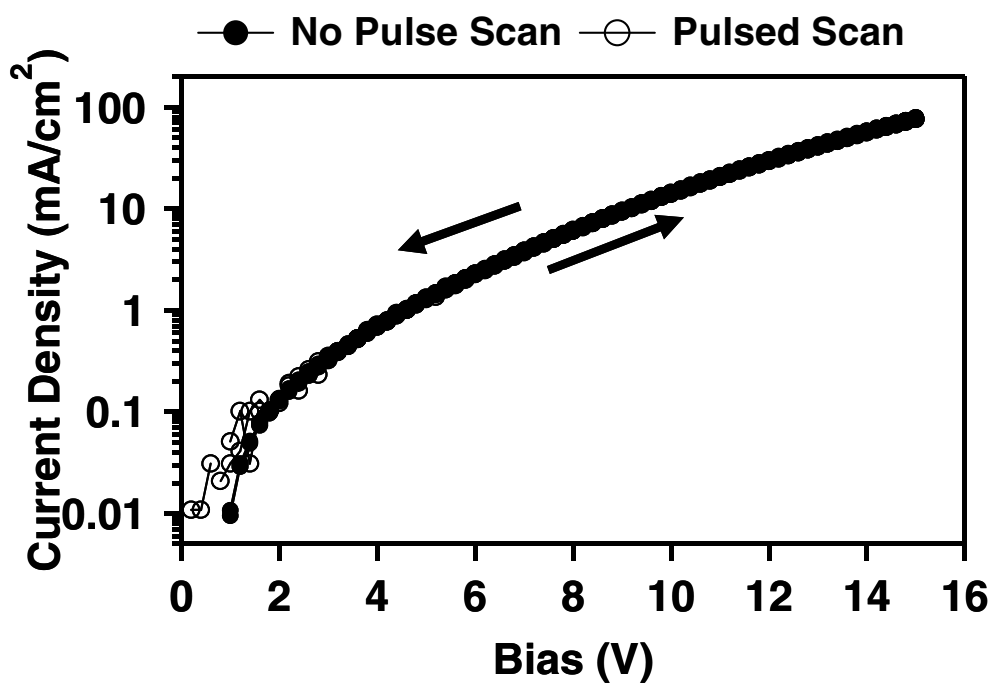

